\title{
ORDER CONVERGENCE AND TOPOLOGICAL CONVERGENCE
}

RALPH E. DEMARR

In a complete lattice it is possible to define a notion of convergence (for arbitrary nets) known as order convergence (o-convergence); for definitions see $[1$, p. 59$]$ and $[3$, p. 65$]$. As a general rule $o$-convergence is not a topological convergence; i.e., the lattice cannot be topologized so that nets $o$-converge if and only if they converge with respect to the topology [2]. It is of interest to know when these two types of convergence coincide. A number of questions could be posed here, but we shall deal only with the question of when a topological space can be suitably embedded in a complete lattice. To make this statement more precise we shall give the following definition.

Definition. A topological space is said to be an $O$-space if it is homeomorphic to a subset $\Omega_{0}$ of a complete lattice $\Omega$ and if every net in $\Omega_{0}$ converges (with respect to the topology for $\Omega_{0}$ ) to a limit in $\Omega_{0}$ if and only if it $o$-converges to this limit. For example, every completely regular Hausdorff space is an $O$-space because it is homeomorphic to a subset of the direct product of unit intervals and if this direct product is partially ordered componentwise, then it becomes a complete lattice in which $o$-convergence is the same as convergence with respect to the product topology. In this paper we prove the following theorem.

Theorem. A topological space is an O-space if and only if it is a regular Hausdorff space.

Proof. If $X$ is an $O$-space, then it is homeomorphic to a subset $\Omega_{0}$ of a complete lattice $\Omega$, where the topology for $\Omega_{0}$ has the properties stated in the above definition. Hence, we only need to show that $\Omega_{0}$ is a regular Hausdorff space. Since limits with respect to $o$-convergence are unique, $\Omega_{0}$ is a Hausdorff space. For each nonempty open subset $U$ of $\Omega_{0}$ define $f(U)=\sup \{\sigma: \sigma \in U\}$ and $g(U)=\inf \{\sigma: \sigma \in U\}$. Then define $S(U)=\left\{\tau: \tau \in \Omega_{0}\right.$ and $\left.g(U) \leqq \tau \leqq f(U)\right\}$. Since $\Omega_{0}$ is an $O$-space, $S(U)$ is closed. It is clear that $U \subset S(U)$.

Now let $\sigma_{0} \in \Omega_{0}$ be any point. We now define the directed set $D$ to be the collection of all pairs $(U, \sigma)$, where $U \subset \Omega_{0}$ is an open neighborhood of $\sigma_{0}$ and $\sigma \in U$. The binary relation $<$ is defined as follows: $\left(U_{1}, \sigma_{1}\right)<\left(U_{2}, \sigma_{2}\right)$ iff $U_{2} \subset U_{1}$. We will now construct a net in $\Omega_{0}$ as

Received by the editors May 28, 1964. 
follows: for each $n=(U, \sigma) \in D$ define $\mu_{n}=\sigma$. It is clear that the net $\left\{\mu_{n}: n \in D\right\}$ converges to $\sigma_{0}$ with respect to the topology for $\Omega_{0}$, hence, it must also $o$-converge to $\sigma_{0}$. From the definition of $o$-convergence we see that $\inf \left\{f(U): U \in N\left(\sigma_{0}\right)\right\}=\sigma_{0}=\sup \{g(U)$ : $\left.U \in N\left(\sigma_{0}\right)\right\}$, where $N\left(\sigma_{0}\right)$ denotes the collection of all open neighborhoods $U \subset \Omega_{0}$ of $\sigma_{0}$.

We will use this latter fact to show by contradiction that $\Omega_{0}$ is regular. If $\Omega_{0}$ is not regular then there exists a point $\sigma_{0} \in \Omega_{0}$ and an open neighborhood $V_{0}\left(V_{0} \subset \Omega_{0}\right)$ of $\sigma_{0}$ such that for every open neighborhood $U$ of $\sigma_{0}$ we have $S(U) \cap V_{0}^{\prime} \neq \varnothing$. ( $V_{0}^{\prime}$ denotes the complement of $V_{0}$.) Therefore, for each $U \in N\left(\sigma_{0}\right)$ one may select $h(U) \in S(U) \cap V_{0}^{\prime}$. Now $\left\{h(U): U \in N\left(\sigma_{0}\right)\right\}$ is a net and since $g(U) \leqq h(U) \leqq f(U)$ for all $U \in N\left(\sigma_{0}\right)$, this net must $o$-converge to $\sigma_{0}$ (recall the results of the previous paragraph). But since $h(U) \in V_{0}^{\prime}$ for all $U \in N\left(\sigma_{0}\right)$, this net cannot converge to $\sigma_{0}$ with respect to the topology for $\Omega_{0}$. This contradicts the fact that $\Omega_{0}$ is an $O$-space; hence, $\Omega_{0}$ must be regular. This completes the proof that every $O$-space is a regular Hausdorff space.

Now assume that $X$ is a regular Hausdorff space. We may assume that $X$ contains infinitely many points, otherwise all is trivial. Let $\mathcal{F}$ be the collection of all closed subsets of $X$ which contain at least two points. Now define three sets $\Omega_{-}, \Omega_{0}$, and $\Omega_{+}$as follows: $\Omega_{-}$and $\Omega_{+}$are sets of ordered pairs of the form $(-1, E)$ and $(+1, E)$, respectively, where $E \in \mathcal{F} ; \Omega_{0}$ is the set of ordered pairs of the form $(0, x)$, where $x \in X$. Then define $\Omega=\Omega_{-} \cup \Omega_{0} \cup \Omega_{+}$. The set $\Omega$ is partially ordered as follows:

$$
\begin{array}{rlll}
(-1, E) \leqq(-1, F) & \text { iff } & F \subset E, \\
(-1, E) \leqq(0, x) & \text { iff } & x \in E, \\
(-1, E) \leqq(+1, F) & \text { iff } & E \cap F \neq \varnothing, \\
(0, x) \leqq(0, y) & \text { iff } & x=y, \\
(0, x) \leqq(+1, E) & \text { iff } & x \in E, \\
(+1, E) \leqq(+1, F) & \text { iff } & E \subset F .
\end{array}
$$

It is easily shown that $\leqq$ is indeed a partial ordering. It is clear that $(-1, X)$ and $(+1, X)$ are the smallest and largest elements, respectively, in $\Omega$.

In general, $\Omega$ is not a lattice, but it can be embedded in a complete lattice $\bar{\Omega}$ (the MacNeille completion); see [1, p. 58]. By the embedding, we can regard $\Omega$ as a subset of $\bar{\Omega}$. Hence, $\Omega_{0}$ can be regarded as a subset of $\bar{\Omega}$. We shall topologize $\Omega_{0}$ so that it is homeomorphic to $X$; this can be done directly since there is a natural one-to-one correspondence between $X$ and $\Omega_{0}$. 
We will now show that a net in $\Omega_{0}$ converges with respect to the topology for $\Omega_{0}$ if and only if it $o$-converges. Let $\left\{\sigma_{n}: n \in D\right\}$ be a net in $\Omega_{0}$ which converges to $\sigma$ with respect to the topology for $\Omega_{0}$. Since $\Omega_{0}$ is a regular Hausdorff space, the collection $N(\sigma)$ of closed neighborhoods of $\sigma$ is a base for the neighborhood system at $\sigma$. Now if the singleton $\{\sigma\}$ is open, then there exists $k \in D$ such that $\sigma_{n}=\sigma$ for all $n>k$; hence, the net $o$-converges to $\sigma$. On the other hand, if the singleton $\{\sigma\}$ is not open, then (putting $\sigma=(0, x))$ we have $\inf \{(+1, E)$ : $E \in N(x)\}=\sup \{(-1, E): E \in N(x)\}=(0, x)=\sigma$, where $N(x)$ is the collection of closed neighborhoods of $x \in X$. Hence, for each $E \in N(x)$ there exists $k \in D$ such that $(-1, E) \leqq \sigma_{n} \leqq(+1, E)$ for all $n>k$. Therefore, the net $o$-converges to $\sigma$.

Now let $\left\{\sigma_{n}: n \in D\right\}$ be a net in $\Omega_{0}$ which does not converge to $\sigma$ with respect to the topology for $\Omega_{0}$. Hence, there must exist a set $E \in \mathcal{F}$ which does not contain $x$, where $(0, x)=\sigma$, such that $x_{n} \in E$ cofinally $\left(\sigma_{n}=\left(0, x_{n}\right)\right)$. Hence, $\tau_{k}=\inf \left\{\sigma_{n}: n>k\right\} \leqq(+1, E)$ for all $k \in D$. Therefore, $\sup \left\{\tau_{k}: k \in D\right\} \leqq(+1, E)$ which means that $\sup \left\{\tau_{k}: k \in D\right\}$ $\neq \sigma$ (recall the definition of the partial order in $\Omega$ and the fact that $x$ does not belong to $E$ ). This in turn means that the net does not 0 converge to $\sigma$. Q.E.D.

\section{REFERENCES}

1. G. Birkhoff, Lattice theory, rev. ed., Amer. Math. Soc. Colloq. Publ. Vol. 25, Amer. Math. Soc., Providence, R. I., 1948.

2. E. E. Floyd, Boolean algebras with pathological order topologies, Pacific J. Math., 5 (1955), 687-689.

3. J. L. Kelley, General topology, Van Nostrand, New York, 1955.

UNIVERSITY OF WASHINGTON 